\title{
MINDFULNESS: ATENCIÓN PLENA EN EDUCACIÓN INFANTIL
}

\author{
Deilis-Ivonne Pacheco-Sanz; \\ Universidad de Valladolid \\ deilisivonne.pacheco@uva.es \\ Alejandro Canedo-García; \\ Universidad de León \\ acang@unileon.es \\ Andrea Manrique Arija; \\ Universidad de Valladolid \\ andreamanriquearija@gmail.com \\ Jesús-Nicasio García-Sánchez \\ Universidad de León \\ jn.garcia@unileon.es
}

Fecha de Recepción: 4 Febrero 2018

Fecha de Admisión: 10 Abril 2018

\section{RESUMEN}

Vivimos en una sociedad donde hasta los más pequeños presentan muchas situaciones de estrés, casi siempre provocadas por los adultos, que no saben cómo afrontarlas. De aquí el interés de investigar sobre las propuestas de intervención que se han realizado para trabajar la atención plena en el aula de educación infantil. Para ello, se ha realizado una revisión de estudios nacionales e internacionales en el campo de la psicología positiva, que permiten comprobar los beneficios que el Mindfulness tiene sobre las personas, específicamente, aquellos que demuestran la utilidad de enseñar a practicar la atención plena a los niños desde edades tempranas. Los programas de intervención diseñados, en torno a este constructo, contemplan actividades en las que los niños son más conscientes de su propio cuerpo, de su respiración, de sus emociones, de las de sus compañeros, etc.; y, evidencian que ahora los niños, a través de la educación emocional, cuentan con una herramienta eficaz que les ayude a afrontar la vida de una manera más consciente y plena, que consigan grandes resultados para su relación consigo mismo y con la sociedad. Se recibieron fondos a través de una ayuda destinada a financiar la contratación predoctoral de personal investigador por la Junta de Castilla y León (EDU/1083/2013), cofinanciada por el Fondo Social Europeo, dentro del Programa Operativo del Fondo Europeo de Desarrollo Regional (FEDER) 2014-2020 de Castilla y León.

Palabras clave: psicología positiva; educación emocional; Mindfulness; educación infantil 


\section{MINDFULNESS: ATENCIÓN PLENA EN EDUCACIÓN INFANTIL}

\section{ABSTRACT \\ Mindfulness: full attention in children's education.}

We live in a society where even the youngest children present many situations of stress, usually provoked by adults, who do not know how to face them. That is why we are interested in investigating the intervention proposals that have been made to work on mindfulness in the classroom for early childhood education. For this purpose, a review of national and international studies in the field of positive psychology has been carried out, which allow to verify the benefits that Mindfulness has on people, specifically, those that demonstrate the usefulness of teaching to practice mindfulness children from early ages. The intervention programs designed, around this construct, contemplate activities in which children are more aware of their own body, their breathing, their emotions, those of their peers, etc .; and, they show that now children, through emotional education, have an effective tool that helps them to face life in a more conscious and full way, which achieve great results for their relationship with themselves and with society. We received funds through a grant to finance the predoctoral recruitment of research staff by the Junta de Castilla y León (EDU/1083/2013), cofinanced by the European Social Fund, within the Operational Program of the European Regional Development Fund (ERDF) 2014-2020 of Castilla y León.

Keywords: positive psychology; emotional education; Mindfulness; child education

\section{ANTECEDENTES}

La psicología positiva es un movimiento innovador, impulsado inicialmente por Martín Seligman, considerado uno de los fundadores de esta rama de la psicología, dedicando la mayor parte de su carrera al estudio de los aspectos positivos del ser humano. (Arguís, Bolsas, Hernández y Salvador, 2012). Fue definida por Seligman y Csikszentmihalyi (citado por Contreras y Esguerra, 2006) como "el estudio científico de las experiencias positivas, los rasgos individuales positivos, las instituciones que facilitan su desarrollo y los programas que ayudan a mejorar la calidad de vida de los individuos, mientras previene o reduce la incidencia de la psicopatología. También Sheldon y King (citado por Contreras y Esguerra, 2006), Io definen como "el estudio científico de las fortalezas y virtudes humanas, las cuales permiten adoptar una perspectiva más abierta respecto al potencial humano, sus motivaciones y capacidades [313]."

Para Linley, Harrington, Stephen, y Wood (citado por Arguís et al., 2012), esta corriente es definida como "el estudio científico del funcionamiento humano óptimo. En un nivel metapsicológico, pretende compensar el desequilibrio en la investigación y la práctica psicológica llamando la atención acerca de los aspectos positivos del funcionamiento y la experiencia humana, e integrándolos dentro de nuestra comprensión de los aspectos negativos del funcionamiento y la experiencia humana. En un nivel pragmático, trata acerca de la comprensión de las fuentes, los procesos y los mecanismos que conducen a éxitos deseables [11]."

La psicología positiva se interesa por temas tan variados como la felicidad o el bienestar subjetivo, las emociones positivas y la inteligencia emocional, el optimismo y la esperanza, la resiliencia y el crecimiento postraumático, las conductas saludables como Mindfulness o el ejercicio físico, la gestión positiva del tiempo, las fortalezas personales, el saboreo, el humor, la capacidad de fluir 0 de mantener el flujo de conciencia, etc. (Alvear, 2015; Bisquerra y Pérez, 2007).

Mayer, Salovey, Caruso y Cherkasski exponían que, en este sentido y dentro de la psicología positiva, tanto la inteligencia emocional como la educación emocional, aportan evidencias de lo que funciona y lo que no. La inteligencia emocional es la capacidad para percibir y expresar las emociones, utilizar las emociones para facilitar el pensamiento, comprender y razonar a través de las emociones y regular las emociones en uno mismo y en los demás. Los conocimientos de la psicología positiva y la inteligencia emocional deben ser difundidos a través de la educación emocional, con el 
objetivo de desarrollar competencias clave para la vida que permitan alcanzar un mayor bienestar (Bisquerra y Hernández, 2017; Hervás, 2009).

\section{MINDFULNESS}

El término "Mindfulness" traducido al español como atención plena, suscita un gran interés en el ámbito científico (Bishop, Lau, Shapiro, Carlson, Anderson, Carmody, Segal, Abbey, Speca, Velting, y Devins, 2004). La definición de este constructo de la psicología positiva va a depender del objeto de estudio y de la práctica. En el caso de Bishop et al. (2004), lo definen como un estado de conciencia que supone "darse cuenta de la experiencia presente con curiosidad, apertura a la experiencia y aceptación." Otras definiciones se encuentran en la tabla 1.

Tabla1.

Definiciones de Mindfulness.

\begin{tabular}{|c|l|}
\hline \multicolumn{1}{|c|}{ Autor } & \multicolumn{1}{|c|}{ Definición } \\
\hline Kabat-Zinn (2007) & $\begin{array}{l}\text { Según Nyanaponika Thera, la atención plena es la } \\
\text { llave maestra infalible y el punto de partida para } \\
\text { el conocimiento de la mente, la herramienta } \\
\text { perfecta y el punto focal para el desarrollo de la } \\
\text { mente, la manifestación más elevada y el punto } \\
\text { culminante de la libertad mental }\end{array}$ \\
\hline Snel (2013) & $\begin{array}{l}\text { La atención plena o Mindfulness, no es otra cosa } \\
\text { que estar conscientemente presente. Queriendo } \\
\text { comprender qué es lo que ocurre, desde una } \\
\text { actitud abierta y amable. Estar "aquí" presente, en } \\
\text { este momento. }\end{array}$ \\
\hline Naht (2015) & $\begin{array}{l}\text { Mindfulness es mantener viva la conciencia en la } \\
\text { realidad del presente }\end{array}$ \\
\hline
\end{tabular}

Fuente: Adaptado de Kabat-Zinn (2007); Snel (2013); Naht (2015).

Por otra parte, la conceptualización global de Mindfulness, presentada por Dimidjian y Linehan (2003) (citado por Parra, Montañés, Montañés y Bartolomé, 2012) , identifica tres cualidades relacionadas con lo que una persona hace cuando realiza esta práctica: i) la observación (el darse cuenta, traer a la consciencia); ii) la descripción (el etiquetado, el reconocimiento); y, la participación.

Asimismo, Parra et al. (2012), identifican tres cualidades relacionadas con el modo en el que una persona realiza estas actividades: i) con aceptación, permitiendo, sin enjuiciar; ii) en el momento presente, con mente del principiante; y, iii) eficazmente.

\section{Emociones y Mindfulness}

En la actualidad, se puede comprobar que la práctica de Mindfulness genera en la mayoría de las personas emociones positivas de diversa índole. Incluso en sujetos que no practican Mindfulness, un nivel alto de Mindfulness tiene relación con un nivel alto en el bienestar subjetivo (Alvear, 2015).

La psicóloga social Fredrickson (2009) (citado por Alvear, 2015), hace una clasificación de las diez emociones positivas más representativas: alegría, gratitud, serenidad y calma, interés-curiosidad, esperanza, orgullo-autoconfianza, diversión, inspiración, asombro-absorción y amor. La mayoría de ellas en menor o mayor grado se pueden generar con la práctica de Mindfulness. 


\section{Los logros y el Mindfulness}

Seligman (citado por Alvear, 2015) añadió el logro como un elemento clave para el bienestar psicológico por su valor intrínseco e independiente de la emoción positiva, de la energía y del sentido. Dentro del concepto de logro, se integrará la consecución de metas cotidianas, de metas vitales y de metas de larga duración.

Aquí aparece la primera discrepancia entre la consecución de la meta (orientada hacia el futuro) y el Mindfulness (orientado al presente). No obstante, si bien son dos constructos (el logro y el Mindfulness) que en principio resultan distantes, hay numerosas investigaciones que avalan una relación entre ambos.

\section{Las relaciones personales y el Mindfulness}

Las relaciones personales positivas es otro de los elementos que Seligman (citado por Alvear, 2015) introdujo en la transición conceptual y metodológica que llevó a cabo al pasar de la Teoría de la auténtica felicidad a la Teoría del bienestar.

Las relaciones positivas entre seres humanos no son solo las que generan satisfacción y bienestar, también se describen como emocionalmente vitales y se caracterizan por la intimidad, el crecimiento personal mutuo y la resiliencia para gestionar adversidades en equipo. Es comprensible que los ámbitos más investigados sean las relaciones de pareja y las relaciones intrafamiliares, al igual que en el Mindfulness que uno de sus objetivos es mejorar la calidad de las relaciones personales, especialmente en el ámbito de la pareja.

\section{Sentido de la vida y Mindfulness}

Alvear (2015), señala que, con el paso de los años, se ha llegado a comprender que el sentido de la vida es una de las claves del bienestar psicológico, tanto desde el punto de vista del bienestar hedonista (se relaciona con la búsqueda de placer y el equilibrio armonioso entre emociones positivas y negativas), como del bienestar eudaimónico (tiene más que ver con el desarrollo de las virtudes y las fortalezas personales y con un crecimiento personal implícito a ello).

Con todo esto, es posible deducir que la práctica de Mindfulness ayuda y genera un aumento tanto del bienestar hedonista como del bienestar eudaimónico.

\section{Flow y Mindfulness}

El flow es un estado subjetivo que las personas experimentan cuando están completamente involucradas en algo, hasta el extremo de olvidarse del tiempo y de la noción de "yo", mientras siguen concentrados en la actividad que realizan. Todo ello, está relacionado con el Mindfulness, especialmente la atención al momento presente, la perdida de la noción de "yo" y la gratificación posterior al hecho de haber experimentado este estado mental (Alvear, 2015).

\section{Saboreo y Mindfulness}

El saboreo implica la autorregulación de las emociones positivas generándolas, manteniéndolas 0 aumentándolas atendiendo a las experiencias positivas del pasado, del presente 0 del futuro, y al igual que sucede con el Mindfulness, la capacidad de saboreo difiere de una persona a otra.

Partiendo de la perspectiva temporal, se ha explorado tres componentes del saboreo: el saboreo mediante la anticipación, el saboreo mediante rememorar el pasado y el saboreo del momento presente. Este último componente es el que se identifica con el Mindfulness. En ambos casos, se cultiva la atención necesaria para experimentar el presente (Alvear, 2015).

\section{Fortalezas personales y Mindfulness}

Las fortalezas personales son capacidades cognitivas, afectivas, volitivas y conductuales que 
suponen los ingredientes psicológicos necesarios para desarrollar las virtudes humanas. El Mindfulness se entiende como un elemento clave y sustancial a la hora de detectar, diagnosticar, implementar y desarrollar diferentes fortalezas personales tanto en una sola persona, como a nivel de intervención grupal. Al igual que, se puede hacer valer el cultivo de las diferentes fortalezas personales para generar una práctica sólida en Mindfulness, convirtiendo ambos constructos en dos elementos clave que se retroalimentan ofreciendo un mayor bienestar al practicante.

También se puede concebir que la mera práctica de Mindfulness supone un desarrollo implícito, sutil y paulatino de varias fortalezas personales. La práctica de la Psicología Positiva llena la vida de las personas de felicidad y bienestar a través del desarrollo de las fortalezas personales (Barahona, Sánchez y Urchaga, 2013).

\section{OBJETIVOS}

La relación de la psicología positiva y el Mindfulness, a partir de los antecedentes presentados, dan prueba de la vinculación de este constructo con diferentes variables dentro del ámbito educativo y psicológico. Es por ello que el objetivo de esta investigación es presentar una revisión de estudios nacionales e internacionales recientes, que demuestren la utilidad de enseñar a practicar la atención plena en el ámbito educativo, específicamente, en la Educación Infantil.

\section{METODOLOGÍA}

Se presenta un estudio descriptivo que se inserta dentro del método de investigación cualitativa que, como toda investigación científica, cumple con dos propósitos: producir conocimiento y teorías (investigación básica) y resolver problemas (investigación aplicada).

\section{RESULTADOS}

\section{Revisión de investigaciones empíricas nacionales e internacionales sobre la práctica de Mindfulness en el ámbito educativo}

De acuerdo a los estudios revisados, el Mindfulness ha ido creciendo considerablemente. Entre los años 1980 y 2000 aparecen 976 referencias a Mindfulness, mientras que entre el año 2000 y 2012 las referencias han aumentado a 12.687, siendo el 84\% de las mismas de los últimos seis años. En este sentido, se considera importante informar que en el año 2010 se editó el primer volumen de una nueva revista llamada Mindfulness. Su principal objetivo es la divulgación del avance científico, la práctica clínica y las teorías referentes a Mindfulness. Está permitido acceder a sus primeros artículos online y se puede hacer desde la página web http://www.springerlink.com/content/121591/?Content+Status=Accepted.

Todo ello ha contribuido a la relevancia que está adquiriendo, a nivel educativo, la práctica del Mindfulness. En diferentes países, durante los últimos años, se han desarrollado proyectos, dentro y fuera de las aulas. Tal es el caso de España con la creación de Escuelas conscientes y Aulas felices (Tébar y Parra, 2015).

En cuanto a los estudios revisados sobre la práctica de la atención plena en el ámbito de la Educación Infantil, los resultados indican que la mayoría se centran en el diseño de programas 0 propuestas de intervención para promover la educación emocional en el aula, a través del Mindfulness (ver tabla 2).

En este sentido, existe suficiente evidencia empírica que demuestra la utilidad de enseñar a practicar la atención plena a los niños desde edades tempranas, de manera que cuenten con una herramienta eficaz que les ayude a afrontar la vida de una manera más consciente y plena (Mañas, Franco, Gil, Gil, 2014; Nhat, 2015; Arrojo, 2015; Tébar y Parra, 2015). 


\section{MINDFULNESS: ATENCIÓN PLENA EN EDUCACIÓN INFANTIL}

Como indica Arguís (citado por Tébar y Parra, 2015), la práctica de Mindfulness en el ámbito educativo puede contrarrestar el estrés diario de forma que se puedan vivir las experiencias de un modo más atento y consciente, permite asimilar la nueva información de manera más serena y relajada, aprendiendo mejor y evitando el riesgo de exceso de información y aporta elementos para el desarrollo personal, social y la promoción del bienestar en las escuelas, complementando así los aprendizajes académicos más tradicionales.

Además, como bien explican Tébar y Parra (2015), el niño de tercero de Educación Infantil posee un gran control corporal, así como una lateralidad completamente definida en la mayoría de los casos, siendo capaz de realizar aproximadamente la totalidad de las actividades que requieren un buen desarrollo tanto de la motricidad gruesa como fina, siempre que hayan tenido la oportunidad de practicar. En referencia al desarrollo cognitivo, Piaget señala que estos niños se encuentran en el estadio preoperacional, regidos por un pensamiento simbólico, prelógico y egocéntrico. Desde la Teoría de la mente, Shaffer y Kipp (2007) (citado por Tébar y Parra, 2015), indican que a los 5 años los niños son capaces de comenzar a comprender que el pensamiento de otras personas no tiene por qué ser igual al suyo propio. La atención va creciendo y es más controlada que en edades anteriores, saben regular la planeación y organización de las actividades y tienen una mejor coordinación de los sentidos, de los procesos de memoria, así como de habla y la audición.

Otros estudios hacen hincapié en la práctica meditativa, demostrando, por una parte, que los niños duermen mejor, se concentran más y se sienten más seguros. Por otra parte, señalan que la comprensión de la naturaleza de la mente no es algo que llegue exclusivamente a través del intelecto, sino que se alcanza a través del adecuado equilibrio entre la comprensión intelectual y la experiencia meditativa. La práctica meditativa en los niños no tiene que ser complicada, larga ni formal (Snel, 2013; Alvear, 2015; Kaiser, 2014).

Tabla 2.

Estudios que demuestran la práctica de la Atención plena o Mindfulness en el ámbito educativo.

\begin{tabular}{|c|c|c|c|c|c|}
\hline Estudio & Participantes & Instrumentos & Foco & $\begin{array}{l}\text { Programa de } \\
\text { intervención }\end{array}$ & Resultados \\
\hline Snel (2013) & $\begin{array}{l}\text { Niños de } 5 \text { a } 10 \\
\text { años y a r sus } \\
\text { padres. }\end{array}$ & $\begin{array}{l}\text { La meditación } \\
\text { mediante } \\
\text { historias y } \\
\text { ejercicios. }\end{array}$ & $\begin{array}{lll}\text { Cómo enseñar a } & \text { los niños a } \\
\text { los a } & \text { meditar y adquirir } \\
\text { una } & \text { atención } \\
\text { plena } & \end{array}$ & $\begin{array}{l}\text { Método de } \\
\text { Mindfulness } \\
\text { desarrollado } \\
\text { por Jon Kabat- } \\
\text { Zinn }\end{array}$ & $\begin{array}{l}\text { Los niños } \\
\text { duermen } \\
\text { mejor, están } \\
\text { más } \\
\text { concentrados } \\
\text { y serenos y se } \\
\text { sienten más } \\
\text { seguros. }\end{array}$ \\
\hline $\begin{array}{l}\text { Mañas, } \\
\text { Franco, Gil } \\
\text { y } \quad \text { Gil } \\
(2014)\end{array}$ & & & $\begin{array}{l}\text { Contribuir a la } \\
\text { introducción } \\
\text { expansión del } \\
\text { Mindfulness en el } \\
\text { ámbito educativo. }\end{array}$ & $\begin{array}{l}\text { Para maestros } \\
\text { /profesores: } \\
\text {-Mindfulness- } \\
\text { Based Wellness } \\
\text { Education. } \\
\text {-Cultivating } \\
\text { Awareness and } \\
\text { Resilience in } \\
\text { Education. } \\
\text {-Stress } \\
\text { Management } \\
\text { and Relaxation } \\
\text { Techniques. } \\
\text { Para los } \\
\text { alumnos }\end{array}$ & \\
\hline
\end{tabular}




\begin{tabular}{|c|c|c|c|c|c|}
\hline & & & & $\begin{array}{l}\text { lestudiantes: } \\
\text {-Inner Kids } \\
\text { Program. } \\
\text {-Inner } \\
\text { Resilience } \\
\text { Program. } \\
\text {-Learning to } \\
\text { BREATHE. } \\
\text {-Mindfulness in } \\
\text { Schools } \\
\text { Proyect. }\end{array}$ & \\
\hline $\begin{array}{l}\text { Lozano y } \\
\text { Vélez } \\
(2008)\end{array}$ & $\begin{array}{l}\text { Alumnos de } 2 \\
\text { años }\end{array}$ & $\begin{array}{l}\text { La } \\
\text { observación } \\
\text { sistemática del } \\
\text { educador. }\end{array}$ & $\begin{array}{lr}\text { Cómo identifican } \\
\text { y r expresan } \\
\text { sentimientos } & \text { y } \\
\text { emociones } & \text { los } \\
\text { niños de } & \text { dos } \\
\text { años, y } & \text { así } \\
\text { mejorar } & \text { sus } \\
\text { habilidades } & \\
\text { sociales. } & \end{array}$ & $\begin{array}{l}\text {-Evaluación } \\
\text { inicial: } \\
\text {-Desarrollo del } \\
\text { proceso de } \\
\text { Enseñanza- } \\
\text { aprendizaje: } \\
\text {-Evaluación } \\
\text { final: }\end{array}$ & $\begin{array}{l}\text { Se } \\
\text { de considera } \\
\text { importancia la } \\
\text { incorporación } \\
\text { de la } \\
\text { educación } \\
\text { emocional en } \\
\text { las aulas del } \\
\text { primer ciclo } \\
\text { de educación } \\
\text { infantil. }\end{array}$ \\
\hline $\begin{array}{l}\text { Nhat } \\
(2015)\end{array}$ & & $\begin{array}{l}\text { Actividades } \\
\text { didácticas. }\end{array}$ & $\begin{array}{l}\text { Manual para que } \\
\text { los adultos usen y } \\
\text { compartan con } \\
\text { los niños, invita a } \\
\text { practicar la plena } \\
\text { consciencia. }\end{array}$ & & \\
\hline $\begin{array}{l}\text { Arrojo } \\
(2015)\end{array}$ & $\begin{array}{l}\text { Niños y niñas de } \\
5 \text { años }\end{array}$ & $\begin{array}{l}\text { Cuestionario } \\
\text { inicial, } \\
\text { evaluación } \\
\text { continua y } \\
\text { final. }\end{array}$ & $\begin{array}{lr}\text { Introducir } & \text { en } \\
\text { prácticas } & \text { de } \\
\text { mindfulness } & \text { o } \\
\text { atención plena } & \text { al } \\
\text { alumnado } & \text { de } \\
\text { Educación } & \\
\text { Infantil } & \end{array}$ & $\begin{array}{l}\text { Basado en la } \\
\text { práctica de } \\
\text { Mindfulness } \\
\text { para la mejora } \\
\text { de la conciencia } \\
\text { plena del } \\
\text { alumnado de } \\
\text { Educación } \\
\text { Infantil. } \\
\text { Metodología } \\
\text { activa, lúdica, } \\
\text { globalizadora y } \\
\text { participativa. }\end{array}$ & $\begin{array}{l}\text { No se ha } \\
\text { puesto en } \\
\text { práctica pero } \\
\text { se espera que } \\
\text { los resultado } \\
\text { sean } \\
\text { beneficiosos y } \\
\text { positivos. }\end{array}$ \\
\hline \multirow[t]{2}{*}{$\begin{array}{l}\text { Parra, } \\
\text { Montañés, } \\
\text { Montañés, } \\
\text { y } \\
\text { Bartolomé } \\
(2012)\end{array}$} & & & $\begin{array}{l}\text { Introducción al } \\
\text { Mindfulness. } \\
\text { Mecanismos } \\
\text { psicoterapéuticos } \\
\text { que subyacen a la } \\
\text { práctica de } \\
\text { Mindfulness. }\end{array}$ & & \\
\hline & $\begin{array}{l}25 \text { alumnos de } \\
\text { entre } 5 \text { y } 6 \text { años }\end{array}$ & $\begin{array}{l}\text {-Semáforo de } \\
\text { las emociones } \\
\text { para los niños. } \\
\text {-Cuestionario } \\
\text { de evaluación } \\
\text { para las } \\
\text { actividades }\end{array}$ & $\begin{array}{lr}\text { La adaptación y } \\
\text { evaluación de una } \\
\text { serie } \\
\text { actividades para } \\
\text { la práctica de } \\
\text { Mindfulness en el } \\
\text { aula de tercer } \\
\text { curso } & \text { de }\end{array}$ & $\begin{array}{l}\text { Metodología } \\
\text { cualitativa, } \\
\text { basada en una } \\
\text { investigación } \\
\text { evaluativa en la } \\
\text { cual prima } \\
\text { principalmente } \\
\text { la labor de }\end{array}$ & $\begin{array}{l}\text { Se logra el } \\
\text { objetivo. }\end{array}$ \\
\hline
\end{tabular}




\begin{tabular}{|c|c|c|c|c|}
\hline $\begin{array}{l}\text { Tébar y } \\
\text { Parra } \\
(2015)\end{array}$ & & & $\begin{array}{l}\text { Educación } \\
\text { Infantil. }\end{array}$ & $\begin{array}{l}\text { evaluar las } \\
\text { actividades } \\
\text { realizadas de } \\
\text { práctica de } \\
\text { atención plena } \\
\text { a través de una } \\
\text { observación } \\
\text { sistemática y de } \\
\text { medidas } \\
\text { cuantitativas } \\
\text { por parte de los } \\
\text { niños } \\
\text { participantes y } \\
\text { de dos jueces. }\end{array}$ \\
\hline $\begin{array}{l}\text { Arguís, } \\
\text { Bolsas, } \\
\text { Hernández } \\
\text { y Salvador } \\
\text { (2012) }\end{array}$ & $\begin{array}{l}\text { Docentes de } \\
\text { Educación } \\
\text { Infantil }\end{array}$ & $\begin{array}{l}321 \\
\text { actividades } \\
\text { para utilizar } \\
\text { en el aula de } \\
\text { infantil. }\end{array}$ & $\begin{array}{lr}\text { Aportar } & \text { al } \\
\text { profesorado un } \\
\text { manual que le } \\
\text { permita conocer } \\
\text { los fundamentos } \\
\text { de la psicología } \\
\text { positiva y le } \\
\text { facilite } \\
\text { estrategias } \\
\text { propuestas } \\
\text { actividades que } \\
\text { pueda utilizar en } \\
\text { las aulas }\end{array}$ & $\begin{array}{l}\text { Perspectiva } \\
\text { amplia, } \\
\text { comprendiendo } \\
\text { diversos } \\
\text { ámbitos de } \\
\text { intervención y } \\
\text { teniendo en } \\
\text { cuenta todas las } \\
\text { edades de los } \\
\text { niveles } \\
\text { educativos } \\
\text { anteriores a la } \\
\text { Universidad }\end{array}$ \\
\hline $\begin{array}{l}\text { Alvear } \\
(2015)\end{array}$ & $\begin{array}{l}\text { Dirigido a } \\
\text { practicantes } \\
\text { experimentados, } \\
\text { para iniciados } \\
\text { así como para } \\
\text { las personas que } \\
\text { quieran mejorar } \\
\text { los aspectos } \\
\text { positivos del ser } \\
\text { humano. }\end{array}$ & $\begin{array}{l}\text { Actividades, } \\
\text { meditaciones } \\
\text { y relajaciones. }\end{array}$ & $\begin{array}{lr}\text { Enseña lo } \\
\text { académico y lo } \\
\text { experimental, lo } \\
\text { teórico y práctico } \\
\text { y establece un } \\
\text { diálogo entre la } \\
\text { sabiduría } \\
\text { universal y } \\
\text { novedosas teorías } \\
\text { científicas. }\end{array}$ & \\
\hline
\end{tabular}

Fuente: Elaboración propia a partir de los estudios revisados.

\section{DISCUSIÓN Y CONCLUSIONES}

En la actualidad, los aspectos racionales y emocionales comienzan a relacionarse asumiendo que no se trata de dos polos opuestos, sino que son sistemas complementarios. Tal y como lo expone Kaiser (2014) en su libro El niño atento, "Io que los padres hacen con sus hijos, la forma en que les hablan y organizan su tiempo, influye en su carácter y les orienta en una determinada dirección. Puede ser creativo, artístico, académico, atlético, espiritual o cualquiera de un amplio elenco de posibilidades, pero el tipo de camino que sea y el lugar hacia el que apunte afectarán a sus hijos a lo largo de su vida. Sea cual fuere el camino que los hijos elijan, las comprensiones que les proporcione la práctica de Mindfulness les ayudarán a elegir un camino con el corazón [24]."

En este sentido, la atención plena o Mindfulness, constituye una forma de aprendizaje muy diferente del enfoque enseñado en la mayoría de las escuelas, que alienta en el niño el deseo de aprender (Parra et al., 2012; Siegel, 2011). 
Por ser una técnica novedosa, con muy poco recorrido tanto en España como a nivel internacional, resulta algo complicado recoger evidencias empíricas. Como ya se ha señalado, la aplicación en los colegios ha sido mínima, pues para poder llevar a cabo una intervención sobre el Mindfulness, el profesorado debe estar formado en ello, y existen pocas formaciones con todos los contenidos que se deben abordar para poder aplicarlo de una forma consciente.

Asimismo, a través de los programas y propuestas de intervención encontrados, se ha podido comprobar cómo se puede llevar a cabo una educación emocional en el aula a través del Mindfulness como constructo de la psicología positiva. Con toda la información y los estudios encontrados sobre la Atención plena o Mindfulness se llega a la conclusión de que se alcanzan muchos beneficios practicándola desde pequeños; por lo que es muy importante que los niños conozcan sus emociones y sepan regularlas, para conseguir un bienestar emocional y un desarroIlo integral (García, 2015; Bueno, 2016).

Como perspectiva futura, y tomando como referencia la revisión teórica aquí presentada, se aspira diseñar una propuesta de intervención para el aula de $3^{0}$ de educación infantil, esperando una serie de resultados positivos y beneficiosos para todos. Es decir, que el niño logre una atención consciente de sí mismo, que aprenda a prestar atención a su respiración, a sus emociones, que conozca técnicas para relajarse, para meditar, para conocerse a sí mismo y para conocer a sus compañeros y así conseguir un clima de bienestar personal y grupal.

Por último, añadir que una de las limitaciones que se encuentran son los escasos estudios empíricos que se han realizado sobre este tema. Con lo cual, a la hora de poner en práctica la propuesta de intervención que se aspira, no permitiría la comparación y el contraste con los resultados que se obtengan.

Otra limitación estaría en la puesta en práctica de una propuesta de intervención de este estilo, pues en los centros educativos tienen que haber docentes conscientes, cuya formación esté orientada hacia cómo formar niños ricos en educación emocional.

\section{BIBLIOGRAFÍA}

Alvear, D. (2015). Mindfulness en positivo. Lleida, España: Milenio.

Arguís, R., Bolsas, A. P., Hernández, S., y Salvador, M. M. (2012). Programa Aula felices. Recuperado de https://psyciencia.com/wp-content/uploads/2013/03/Aulas-felices.pdf

Arrojo, S. (2015). Programa de Mindfulness aplicado a educación infantil (Tesis de pregrado). Universidad de Granada, España.

Barahona, M.N, Sánchez, A y Urchaga, J.D. (2013). La Psicología Positiva aplicada a la educación: el programa CIP para la mejora de las competencias vitales en la Educación Superior. Revista de Formación e Innovación Educativa Universitaria. Recuperado de http://refiedu.webs.uvigo.es/Refiedu/Vol6_4/REFIEDU_6_4_5.pdf

Bishop, S., Lau, M., Shapiro, S., Carlson, L., Anderson, N., Carmody, J., Segal, Z., Abbey, S., Speca, M., Velting, D., y Devins, G. (2004). Mindfulness: A Proposed Operational Definition. Clinical Psychology: Science and Practice, 11(3), 230-241. Recuperado de file:/// C: / Users / Usuario/DownIoads/Bishop_e t_al - 2004 Clinical_Psychology\%253A_Science_and_Practice\%20(1).pdf

Bisquerra, R, y Hernández, S (2017). Psicología positiva, educación emocional y el programa aulas felices. Recuperado de http://www.papelesdelpsicologo.es/pdf/2822.pdf

Bisquerra, R. y Pérez, N. (2007). Las competencias emocionales. Facultad de pedagogía, universidad de Barcelona, España.

Bueno, L. (2016). Atención plena en el aula de educación infantil. (Tesis de pregrado). Universidad de Extremadura, Badajoz, España. 
Contreras, F, y Esguerra, G (2006/05/09). Psicología positiva: una nueva perspectiva en psicología. Revista diversitas. Recuperado de http://pepsic.bvsalud.org/pdf/diver/v2n2/v2n2a11.pdf

García, M. (2015). Mindfulness para la autorregulación emocional en las aulas del primer ciclo de educación infantil. (Tesis de pregrado). Universidad de Sevilla, España.

Hervás, G. (2009). Psicología positiva: una introducción. Revista interuniversitaria de formación del profesorado. Recuperado de http://m.aufop.com/aufop/uploaded_files/articulos/ 1258587094.pdf

Kabat-Zinn, J. (2007). La práctica de la atención plena. Barcelona, España: Kairós.

Kaiser, S. (2014). El niño atento. Bilbao, España: Desclée de Brouwer.

Lozano, J. y Vélez, V. (2008). La educación de emociones básicas en niños y niñas de dos años. Recuperado

de http://www.um.es/documents/299436/550133/VELEZ+ORTIZ,+ELENA+y+LOZANO+MARTINEZ,+JOSEFINA.pdf

Mañas, I. Franco, C. Gil, M.D. Gil, C. (2014). Educación consciente: Mindfulness (atención plena) en el ámbito educativo. Educadores conscientes formando a seres humanos conscientes. Recuperado de http://www.psicologiamindfulness.es/assets/pdfs/39884-20.pdf

Nhat, T. (2015). Plantando semillas. Barcelona, España: Kairós.

Parra, M., Montañés, J. Montañés, M. y Bartolomé, R (2012/12/12). Conociendo Mindfulness. Revista de la Facultad de Educación de Albacete. Recuperado de https://www.revista.uclm.es/index.php/ensayos/article/view/130/112

Siegel, R.D. (2011). La solución Mindfulness. Bilbao, España: Desclée de Brouwer.

Snel, E. (2013). Tranquilos y atentos como una rana. Barcelona, España: Kairós.

Tébar, S. y Parra, M. (2015/12/28). Practicando Mindfulness con el alumnado de tercer curso de educación infantil. Revista de la Facultad de Educación de Albacete. Recuperado de file:///C:/Users/andrea/Downloads/912-3953-1-PB.pdf

Villegas, M. (2006). Mindfulness y psicoterapia. Revista de psicoterapia. Recuperado de http://roderic.uv.es/bitstream/handle/10550/56496/114802.pdf?sequence=1 